\title{
Procesos de articulación colectiva y los múltiples trazos de su politicidad
}

\author{
Cristián Muñoz e David Romero*
}

http://dx.doi.org/10.22409/poiesis.1829.041055

\begin{abstract}
RESUMEN: El siguiente texto es un segmento del libro La puesta a prueba de lo común, en el cual se aborda la producción artística contemporánea en la ciudad de Concepción, Chile. Particularmente, profundizamos en algunas experiencias de trabajo colectivo que, a nuestro juicio, nos acercan a la dimensión política de aquello que denominamos como "procesos de articulación colectiva" en el campo del arte contemporáneo. Desde nuestra perspectiva, el trabajo editorial así como las producciones que indagan en la "proyección social de la práctica artística" han aportado una reflexión relevante respecto de la relación entre arte y esfera pública dentro del circuito artístico de la ciudad de Concepción, explorando la dimensión política de las manifestaciones artísticas de carácter colectivo.
\end{abstract}

PALABRAS-CLAVE: articulación colectiva, política, arte

ABSTRACT:The following text is a segment of the book La puesta a prueba de lo común, which deals with contemporary artistic production in the city of Concepción, Chile. In particular, we delve into some experiences of collective work that, in our opinion, bring us closer to the political dimension of what we call "processes of collective articulation" in the field of contemporary art. From our perspective, the editorial work as well as the productions that investigate the "social projection of the artistic practice" has contributed a relevant reflection regarding the relationship between art and public sphere, within the artistic circuit of the city of Concepción, exploring the dimension politics of artistic manifestations of a collective nature.

KEYWORDS: collective articulation, politics, art

\footnotetext{
*Cristián Muñoz y David Romero son Licenciados en Arte de la Universidad de Concepción e investigadores independientes de la región del Biobío, Chile. Han realizado un sostenido trabajo dedicado a la reflexión crítica en torno a la producción artística contemporánea, el que se ha visto concretado en diversas publicaciones.
} 
El siguiente texto es un segmento del libro La puesta a prueba de lo común, en el cual se aborda la producción artística contemporánea en la ciudad de Concepción, Chile. Particularmente, profundizamos en algunas experiencias de trabajo colectivo que, a nuestro juicio, nos acercan a la dimensión política de aquello que denominamos como "procesos de articulación colectiva" en el campo del arte contemporáneo. Desde nuestra perspectiva, el trabajo editorial así como las producciones que indagan en la "proyección social de la práctica artística" han aportado una reflexión relevante respecto de la relación entre arte y esfera pública dentro del circuito artístico de la ciudad de Concepción, explorando la dimensión política de las manifestaciones artísticas de carácter colectivo.

Articulación colectiva, política, arte

\section{Lo litigioso y la configuración de esfera pública dentro del espacio artístico}

Un punto sustancial en esta investigación dice relación con la tendencia a la conexión e interacción entre los productores visuales, y el surgimiento de lo que en términos generales podríamos catalogar como "formas colectivas" dentro del espacio artístico en la ciudad de Concepción.

Antes de profundizar en la heterogeneidad de este fenómeno, es preciso advertir que un giro asociativo, vinculado con rutinas de autogestión y emprendimiento, se encuentra ya internalizado en la concepción hegemónica y corriente del trabajo artístico contemporáneo. En otras palabras, lo asociativo pertenece a un universo de nociones y modos de hacer más o menos estandarizados, cuya existencia los agentes locales reconocen nítidamente. Esta modalidad de trabajo se debate tras las lógicas de producción y consumo cultural globalizado (y la consecuente precarización laboral propia de esta etapa), dando la impresión de que el filo crítico de lo colectivo ha perdido consistencia en favor de una normalización y/o neutralización generalizada.

Esta percepción se agudiza debido al abandono de su vinculación con proyectos orientados a la conquista de mayores grados de autonomía, medios de autovalorización o experimentación micropolítica. Así, es notorio el papel mermado que en el escenario actual le cabe a una versión más compleja del fenómeno colectivo, que correspondería llamar "articulación colectiva". 
De cara al giro asociativo que define la pragmática del trabajo cultural contemporáneo, la noción de "articulación colectiva" no se limita a designar el solo ejercicio de trabajar en forma agrupada, sino que intenta dar cuenta de amplias y heterogéneas interacciones en las cuales asomaría una dimensión "instituyente" de renovadas prácticas y acciones. Lo que allí se instituye es, también, un campo de reconocimiento y valoración propio, que describiríamos como una especie de "esfera pública del arte", esto es, un espacio común tanto de discusión como de acción, en el que la interacción y la cooperación mantienen en algún grado su "carácter excedente" respecto de la productividad normalizada' que hoy las convoca abiertamente.

Desde nuestra perspectiva, dicho carácter excedente puede ser reconocido en las formas más elaboradas y complejas de las articulaciones colectivas, al hacer emerger la politicidad que deriva de la apertura, la heterogeneidad y la conflictividad. En este sentido, consideramos relevante remarcar ciertos escenarios o momentos "litigiosos" que ponen de manifiesto la confrontación y el debate, entendiendo que solo a través del disenso se deja reconocer el territorio como verdadero espacio común del hacer. Precisamente, lo litigioso y la conflictividad definen el carácter excedente de la cooperación, en la medida en que dicen relación con el carácter indeterminado y no prefijado de los efectos de las interacciones, propiedades que se contraponen a la norma de la eficacia que manda la productividad normalizada y que pueden ser identificados como atributos sustantivos en la conformación de esfera pública. Es decir, la indeterminación se opone al imperativo de eficiencia y efectividad que configura las formaciones colectivas mejor ajustadas a las demandas de una organización hegemónica del ámbito artístico. La indeterminación, como resistencia al control y la eficiencia exhaustivos exigidos en el margen del proyecto o del plan, sugiere cierto grado de cooperación excedente, no circunscrita de manera directa o evidente al espacio de la productividad de mercado: cooperación excedente que es condición y/o secuela de la conformación de esfera pública.

En igual sentido, lo litigioso correspondería a una propiedad inherente a la constitución de esfera pública, en tanto señala un espacio de interacción y comunicación fundamentalmente disensual, que no puede ser identificado con el calce armonioso o la unión mecánica de las partes en un aparato productivo neto ${ }^{2}$. Así, momentos litigiosos pueden ser tenidos como índices de formas de composición colectiva que reservarían la alternativa de sortear el influjo de las lógicas y las prácticas que neutralizan la politicidad de la interacción y que se someten a la norma de la productividad o a la ética del consenso. 


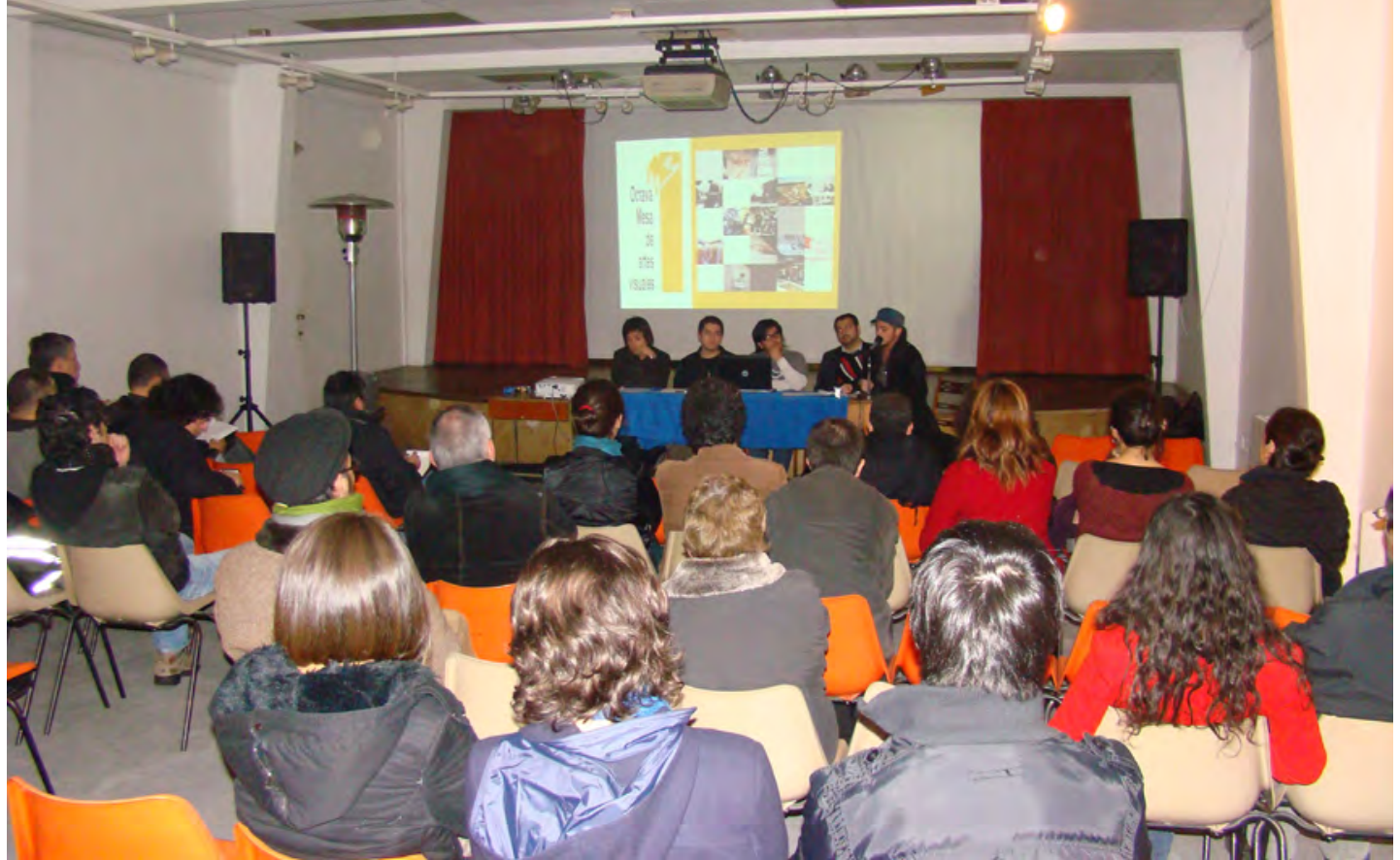

Asamblea organizada por la Octava Mesa de Artes Visuales, 27 de mayo de 2008. La asamblea se convocó en las dependencias de la Corporación Cultural Alianza Francesa de la ciudad de Concepción; en ella, Octava Mesa presentó su proyecto de trabajo con el objetivo de que fuera discutido públicamente por la comunidad artística penquista, así mismo, invitó abiertamente a los agentes a sumarse a la agrupación.

(Fotografías archivo Mesa8)

\section{Trabajo editorial}

Un momento en el que se expone el desarrollo de interacciones complejas, cruzadas por lo conflictivo y lo litigioso, está constituido por la coyuntura que supuso el surgimiento de iniciativas editoriales independientes. A mediados de la década pasada, la apremiante emergencia de contemporaneidad artística exigida a nuestro medio, en parte producto de la intervención de políticas culturales que apuntaban a ese objetivo, puso en juego distintas posiciones a la hora de dar con la especificidad de la escena local y establecer las perspectivas para propiciar sus rasgos de contemporaneidad. Es en dicho contexto que aparece Revista Plus. Soporte de Inscripción Contingente el año 2005, como una plataforma editorial que procuró abordar críticamente un proceso en el cual se intentaban implementar pragmáticos modelos de 
sustentabilidad y desarrollo dentro del espacio artístico regional. El gesto dado por Revista Plus puede ser apreciado como un "tomar la palabra" dentro de un horizonte que, a juicio de sus responsables, implicaba asumir

los inexorables roces y fricciones propios de toda recomposición de escena, cuestiones que precisamente son las que le otorgan su valor, en tanto procuran generar una escena de escritura que no se contenga en la confrontación de puntos de vista y en la generación de agenciamentos que tramen su propia política inscriptiva, en efecto, todo esto no tiene relación ni con la producción de objetos aislados ni con la delimitación de autonomías, sino con la operación de plataformas reflexivas, tramadas y agenciadas, que enriquezcan, por la vía de la interrogación y la transferencia enunciativa, el ahora circuito "abierto" de producción artística penquista. (ROMERO, 2005, p. 5)

En este sentido, junto a la intención de activar escenarios para la discusión y la confrontación, Revista Plus hizo explícita una "disposición interactiva y colaborativa" como clave para la producción artística local y como cifra de un procedimiento crítico orientado a expandir el rango de interlocución y discusión entre los agentes. En definitiva, se esbozaba la posibilidad de levantar una esfera pública del arte local en donde se sometieran a discusión las diversas problemáticas que concernían a los agentes. Esta disposición interactiva y colaborativa podía ser apreciada como un atributo a la hora de favorecer y fomentar una capacidad de cambio y redefinición que respondiera activamente a una época en que, precisamente, el arte más vivo se asumía privado de certezas, pero exigido a su vez de concretar acciones de rango instituyente.

Esta preocupación por la forma pública de constitución del sentido del arte permitiría apreciar en una propuesta artística la simultaneidad y el mutuo compromiso entre el trabajo experimental de constitución de un tipo de colectividad y la instauración de un objeto o acción que, dentro de esa colectividad, se distingue como arte. Lo anterior es lo que, en definitiva, permite la configuración de una "esfera pública del arte" que no se identifica con la especificidad de un público académico o de diletantes, filósofos, estudiantes, etc., sino que, por el contrario, se entiende como colectividad y margen de acción que involucra todas aquellas instancias en las que se potencian la interacción y la cooperación. Así entonces, esta clase de experiencias hacen visible un fenómeno de interdependencia y marcan los mecanismos que posibilitan la decantación de mínimos convenios, pero a su vez insistiendo en conservar la impresión de la apertura e indeterminación que conlleva lo público. 
El trabajo de articulación entre agentes configura una puesta en común, pero desde un plano inicial de confrontación e intercambio que interrumpe la inclinación a adoptar a priori posiciones cerradas de tono esencialista. Más bien, habría que pensar la articulación colectiva como un escenario pertinente para moverse dentro de un entorno desestabilizado y desestabilizador, litigioso, que tiende a caracterizar a la práctica artística como una entidad exigida a establecer definiciones transitorias o en permanente redefinición. Es decir, si bien es cierto las relaciones se inscriben como ámbito imprescindible en la definición de la prácticas o de un régimen de identificación del arte, son al mismo tiempo responsables de la condición siempre provisional de éstas.

\section{Articulación colectiva y proyección social de la práctica artística}

Otra consideración relevante con respecto al carácter litigioso de la articulación colectiva se relaciona con la persistencia de una pregunta por la proyección social del arte y la crítica, a lo cual parece unirse el carácter contextual presente en determinadas prácticas artísticas locales.

Reconociendo lo acotado de las experiencias acontecidas dentro de la línea que indaga problemáticamente en la proyección social de la práctica artística, igualmente parecen haber determinado una cualidad específica de nuestro medio en comparación con la producción artística desarrollada en otros circuitos regionales ${ }^{3}$. Nos atreveríamos a decir, acaso, que, en estrecho contacto con este plano de la producción local, se han elaborado las más interesantes experimentaciones asociadas a la articulación colectiva, en tanto en éstas la propensión a reconfigurar el marco artístico instituido otorgaría a lo colectivo una capacidad de implicación que resitúa críticamente nociones como las de contexto y comunidad. En este itinerario, situamos el caso particular de la galería de la Corporación Cultural Balmaceda Arte Joven sede Biobío, la que, en su apertura, estableció un criterio curatorial orientado a impulsar propuestas artísticas que exploraran abiertamente la relación con el contexto (privilegiando iniciativas colectivas) y que plantearan estrategias de vinculación con el barrio en el que se sitúa Balmaceda Arte Joven ${ }^{4}$.

Dentro de la primera convocatoria de la galería bajo este eje curatorial, se seleccionaron propuestas que, en su mayoría, plantearon una metodología de trabajo colectivo. En lo que respecta a la relación con el contexto, podemos mencionar el trabajo de la Octava Mesa de Artes 
Visuales con "P(L)anificación", así como la obra "Paisaje de la Transparencia", realizada por un colectivo de dibujo conformado por alumnos del Departamento de Artes Plásticas de la Universidad de Concepción. Ambos trabajos, en efecto, recogieron el argumento planteado por la convocatoria. El primero de ellos lo hizo a partir de un proceso de diálogo con personas de la población aledaña a Balmaceda Arte Joven, con el objetivo de cotejar miradas e intercambiar opiniones en torno a la función y la relación que establecería el centro cultural con la comunidad. La acción culminó con una actividad que coordinaron los integrantes del colectivo y pobladores del sector: se instaló un horno industrial dentro de la galería para cocinar y compartir pan, entendido como un acto de implicación que permitiese pensar en futuras políticas que entrelazaran a la comunidad, el centro cultural y los productores visuales. Por su parte, "Paisaje de la transparencia" consistió en un trabajo colectivo de dibujo sobre los muros de la galería, el cual plasmaba la imagen del paisaje que rodeaba al centro cultural. Así, la propuesta de los alumnos de la escuela de arte indagó en la reconfiguración de la mirada sobre el paisaje, llevando éste al interior de la galería y haciendo de la misma un espacio transparente, remarcando que el contexto constituía una proyección problemática e ineludible. Con todo, durante al menos tres años luego de ser lanzada su primera convocatoria, la galería del centro cultural Balmaceda Arte Joven potenció intensamente propuestas que establecían algún tipo de vinculación con la contingencia, es decir, obras o acciones que dialogaban conflictivamente con la realidad ineludible dentro de la cual se desplegaba la producción artística local.

A la luz del proceso del que da cuenta este periodo de la producción artística en Concepción, ¿qué clase de dilemas es posible reconocer, sobre todo cuando nos vemos remitidos a experimentaciones en cuya proyección se trasciende o conflictúa lo meramente estético?

Un concepto apropiado para abordar el problema dice relación con lo que llamaremos la investigación problemática sobre la proyección social de la práctica artística contemporánea. Es decir, en principio se asume que el nexo entre práctica artística y comunidad está cruzado por la conflictividad, en tanto de ningún modo se le puede atribuir al arte, como institución, una presencia inmediata que garantice su carácter natural o necesario. Además, es desplazada aquella noción ideal de comunidad en donde al arte le cabría un papel en la armonización de la identidad colectiva y la recuperación del factor comunicativo. Por el contrario, desde una perspectiva que atiende a la "falla" inscrita en la constitución misma de la comunidad - que nunca es completa ni armónica, sino atravesada por la conflictividad -, podríamos afirmar que 


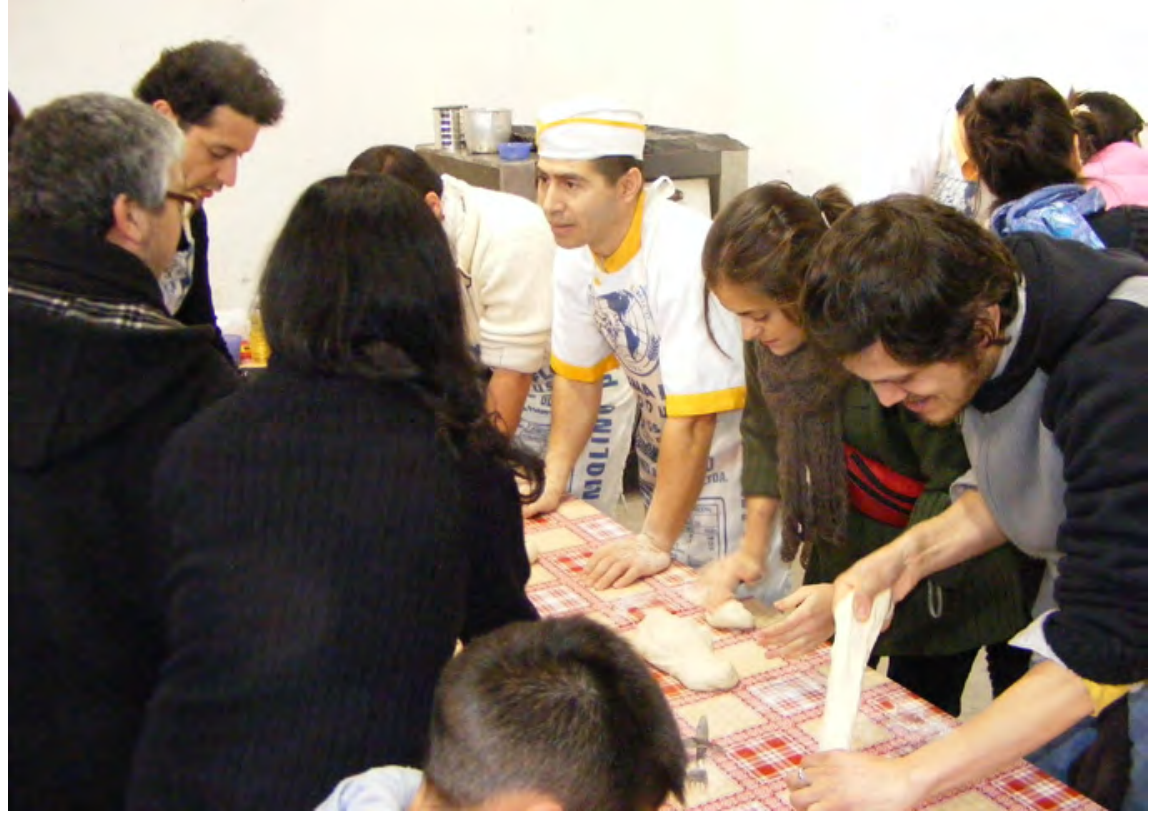

P(L)anificación (2008), Octava Mesa de Artes Visuales. Propuesta realizada en el marco de la convocatoria lanzada por la galería del Centro Cultural Balmaceda Arte Joven, sede Biobío (Concepción). Consistió en un proceso de diálogo con la comunidad que acoge el centro cultural (Tucapel Bajo), el cual culminó con una acción de carácter colectivo. Se instaló un horno industrial dentro de la galería para cocinar y compartir pan, entendido como un acto que permitiese pensar en futuras políticas que entrelazaran a la población, el centro cultural y los productores visuales.

(Fotografías archivo Mesa8)

la curatoría planteada por la galería de Balmaceda Arte Joven dio cuenta de la (im)posibilidad inscrita en el ejercicio de tramar relaciones con el contexto, con la comunidad, en un proceso marcado por la tensión entre autonomía y proyección social. Por otro lado, aquellas relaciones complejizan el uso de los recursos representacionales por parte del espacio artístico, de cara a la evidencia sobre el papel que le cabe a éstos en la producción de la propia comunidad.

Evidentemente, se connota así que nada de lo que hemos venido analizando puede ser situado bajo la bien pensante noción de una "democratización del arte" o de la retórica que celebra lo "participativo" como valor esencial de la producción artística contemporánea. Por el contrario, intentamos dar cuenta de un trabajo en la producción cualificada y contingente de relaciones más allá del espacio material de la galería, las que indudablemente pueden resultar complejas o friccionadas, pero que, sin embargo, permiten abrir un espacio de interacción, efectuando intercambios con el territorio. Todo ello supone una generación de significaciones 
e identificaciones de muy amplio espectro, ya que éstas definen recíprocamente tanto al espacio dado por el contexto y la comunidad como también al propio espacio de la práctica artística, dando lugar a lo que llamaríamos un nuevo y provisional régimen de identificación del arte. Luego, el concepto de "articulación colectiva" exhibe aquí su doble connotación, según la cual, por un lado, describe la densidad y diversidad de las interacciones suscitadas por ciertas prácticas, y por otro, refiere a la complejidad inherente a éstas, por cuanto reconoce la simultaneidad y la reciprocidad de la constitución de las entidades que se articulan. En definitiva, la articulación colectiva, más allá de la amplitud del espectro de las relaciones, precisa la complejidad de un modelo de comprensión en el que se registra la conformación simultánea de las entidades: arte y comunidad.

En el espacio de relaciones que consideramos, la práctica artística entra a batallar en el escenario de una producción simbólica difusa, misma que hoy, se entiende, juega un papel ostensible y corriente en la definición de todo contexto y comunidad. En este sentido, podríamos afirmar que la indagación en torno a la proyección social y la implicación con el contexto no excluye el retorno de una definición, provisional, de la identidad artística. Es decir, las relaciones que se experimentan hacen parte insoslayable de una política de auto-valoración y, además, la implicación no es ajena a un proceso instituyente y a la constitución de cierta especificidad de lo artístico. De esa forma, emerge la condición ambivalente y contaminada del terreno en que se encuentran arte y comunidad, para, simultánea y recíprocamente, producir el colapso de identificaciones y asimismo nuevas alternativas para ésta.

\section{Diferencia y comunidad política}

Pero entonces, ¿cuál es la idea de comunidad que subyace a las prácticas que investigan problemáticamente la proyección social sobre una base de la articulación colectiva? Justamente, hablamos de una investigación problemática en tanto refiere a una comunidad disensual, alejada de las nociones de armonía, completitud, y transparencia. Es decir, la investigación problemática de la proyección social de la práctica artística y los procesos de articulación colectiva que se le asocian comunican con la naturaleza política de la comunidad; luego, se admite que esta última se encuentra cruzada por la imposibilidad, la negatividad y la conflictividad, quedando por ende sujeta a la función constitutiva de la representación. De allí que la tensión y el 
conflicto no puedan ser soslayados u omitidos, precisando más bien de un espacio eminente para empujar la movilidad y el cambio.

Por efecto de lo antes señalado, los procesos de articulación colectiva que ponen en juego la participación de productores artísticos y de otros agentes que, de modo algo apresurado, reconoceríamos como parte de "la comunidad", lo hacen bajo el entendido de la cualidad singular, discreta y no universalizable de las composiciones o arreglos que supone en cada caso la articulación. Así, la articulación se asemeja efectivamente a lo que puede ser identificado como "comunidades imaginadas", en tanto situaciones excepcionales o experimentales de interacción. Es aquel espacio discreto de la articulación el que decimos puede tener efecto en cuanto a la significación o representación de un contexto o comunidad, al tiempo que favorece el alcance instituyente de determinadas prácticas artísticas.

Dicho lo anterior, cabe hacer notar lo que se podría designar como "el carácter menor" de las significaciones y representaciones producidas, como también de las prácticas que genera la articulación colectiva. Es decir, si bien la articulación determina la posibilidad de establecer sentidos compartidos, tanto por lo que toca a la producción de representaciones como a la institución de prácticas, lo hace de manera contingente y provisional. Por lo tanto, la capacidad de significar y de instituir que concentra una articulación se halla distante de producir fenómenos ostensibles, de presencia nítida e inmediata, que complazcan la demanda de "impacto social" tan internalizada en nuestro medio.

Las propiedades políticas de la articulación colectiva en su nexo con la proyección social de la práctica artística, dicen relación con la capacidad de producir significaciones diferenciales en base a procesos tramados que no carecen de conflicto, pudiendo así oponer su signo a la generalización de la norma ética que coloca la universalidad y el consenso como principios y horizontes de legitimidad. Entonces, la idea de comunidad política que sirve como referencia para el análisis del nexo entre articulación e investigación problemática en torno a la proyección social de la práctica artística, establece que

[lo] político no se juega en una de las partes en contienda sino que representa el propio espacio de tensión entre las partes en disputa. Por ello, hay política cuando se mantiene viva dicha tensión. En otros términos, es crear un espacio de polémica, de litigio, de desacuerdo, para convocar y confrontar las identidades. (PANOTTO, 2011, par. 5) 
De acuerdo a lo anterior, se vuelve a apreciar el desajuste insoslayable entre práctica artística y comunidad, aspecto que deriva de la in-completitud y la definición siempre contingente de ambos espacios en una relación que, reconocemos, es simultánea y recíprocamente constitutiva. Es decir, mediante la articulación colectiva, como medio que posibilita la reunión y relación tensa de diferentes entidades, tendría lugar un tipo de des- identificación que, especialmente en lo que respecta al contacto entre arte y comunidad, permite a estos re-significarse. De ahí nuevamente el paralelo entre la articulación (como medio de una investigación problemática sobre la proyección social del arte) y la comunidad política, ya que lo que ésta produce es un espacio de cuestionamiento que favorecería la constante movilidad.

Al poner en foco el trámite de obtención de cierto grado de reconocimiento y valorización social de las prácticas que exploran en la proyección social del arte, éste puede ser visto como la constitución de unas bases de valorización rudimentarias, además de ser un proceso expuesto y completamente explícito. Sin embargo, es posible proponer que aquí la contingencia, el carácter explícito y rudimentario no son deficiencias atribuibles a un estadio inicial, susceptible de alguna superación, sino manifestaciones de aquella politicidad que condiciona y cualifica el espacio construido de las identificaciones. De tal forma, la práctica artística que desarrolla una investigación problemática en torno a su proyección social, con base en una articulación colectiva, adopta el carácter de una presencia diferencial cuyo valor no puede zanjarse de manera rotunda o estable, ni menos consensuarse plenamente, de modo que su significación y valor constituyen motivos vivos de incertidumbre y confrontación; es decir, remiten de forma muy marcada al espacio de la significación y el valor como campo de litigio.

En definitiva, la correspondencia entre el hacer articulado y los perfiles de la comunidad política da lugar al cuestionamiento de la idea de consenso y sus expresiones específicas en el ámbito del arte, ya sea en lo que toca a la definición de unas políticas culturales, ya sea en cuanto a la aceptación sin cuestionamiento de ciertas hegemonías o la reproducción acrítica de tendencias que remarcan la funcionalidad del arte para la producción de relaciones (estética relacional). Así, dentro de ese marco, podemos valorar la serie de experiencias que hasta aquí han sido observadas: la asamblea convocada por la Octava Mesa de Artes Visuales, el trabajo editorial realizado por ciertas publicaciones independientes o la labor efectuada por la galería de Balmaceda Arte Joven durante sus primeros años de funcionamiento. En estas iniciativas se manifiesta el propósito de colocar la potencia de lo colectivo al servicio de una 
investigación y una experimentación que exceda los compartimentos habituales de la producción artística local.

\section{El viraje ético del sistema-arte versus la especificidad de las prácticas locales}

Se observa que la relación entre práctica artística, contexto y/o comunidad, se encuentra suspendida sobre una oscilación que resulta problemática y que, desde una perspectiva crítica, claramente sería deseable evitar; esto es, la caída en un proceder "asistencialista" que hace del arte una esfera privilegiada de reparación social o dotada de alguna especial eficiencia en la resolución de los problemas de determinado contexto o comunidad. Es necesario apuntar este problema, en tanto se aprecia que el desarrollo de procesos o prácticas artísticas en contexto, guiadas por una investigación problemática de la proyección social, es o ha sido preocupación de un número importante de productores locales. Por otro lado, apuntarlo nos permite indagar aún más en la cuestión de "lo político" y en cómo éste es puesto en obra a través de propuestas artísticas que se vinculan a medios y procesos que no pertenecen, necesariamente, al dominio del sistema arte y su orden de validaciones.

Entonces, planteada la disyuntiva, ¿cómo determinaríamos la frontera entre una práctica artística articulatoria y por lo tanto portadora de un "disenso", de otra que se coordina con las ideas de reparación y consenso?

En su libro El viraje ético de la estética y la política, Jacques Rancière establece la oposición entre la comunidad política y la comunidad ética. Esta última ha logrado instalarse como modelo hegemónico de convivencia, amparando los conceptos de igualdad, inclusión universal y consenso. La comunidad ética es, por tanto, una comunidad despolitizada:

[El] consenso significa un modo de estructuración simbólica de la comunidad, que evacua el corazón mismo de la comunidad política, es decir, el disenso. [...] La comunidad política es así tendencialmente transformada en comunidad ética, es decir, en comunidad de un solo pueblo, donde todo el mundo supuestamente cuenta. (RANCIÈRE, 2005, p. 28)

En la comunidad ética, todo el mundo cuenta, señala Rancière. Sin embargo, ésta reacciona de modo violento cuando el excluido llega a radicalizar sus acciones y demandas, es decir, la comunidad ética oscilaría entre unas políticas de la proximidad y unas políticas de la violencia 
más extrema cuando su acción "terapéutica" sobre el conflicto se ve en problemas. Dentro del campo cultural, lo anterior ha modelado ciertas tendencias que se solazan en la composición de una convivencia armónica, más "auténtica" en tanto se supone opuesta a las sujeciones propias de la carga cotidiana. Así por ejemplo, sustentada en la celebración liberal de la diferencia y la pluralidad, la estética relacional hace suyo el vaciamiento de toda conflictividad o antagonismo que pueda friccionar el tan anisado "estar juntos". Pero ello no hace sino mostrar la forma de una manifestación realmente autoritaria, que dista mucho de un ejercicio más denso de lo político, sobre todo si pensamos que "una sociedad democrática es aquella en que se mantienen -en lugar de borrarse- las relaciones de conflicto. Sin antagonismo sólo existe el consenso impuesto propio del orden autoritario, una supresión total del debate y la discusión, nociva para la democracia". (BISHOP, 2005, par. 6)

Siguiendo esta línea de análisis, el sistema artístico contemporáneo se haría parte del viraje ético denunciado por Rancière, cuando se consagra a reafirmar la composición del lazo social y a neutralizar lo político bajo una celebración de la diferencia que no da espacio a la incertidumbre o la confrontación. Por otra parte, la mayoría de las tendencias artísticas que se pliegan a dicha retórica son amparadas por una importante red institucional que funciona como su soporte de legitimación, circulación y valoración; todo lo cual configura un orden que, contrariamente a lo que quiere hacer ver, normaliza la diferencia y la politicidad bajo pragmáticos criterios de consumo y exhibición.

\section{Notas}

1 Es preciso explicitar la paradoja de hacer alusión al "carácter normal" de la producción, en tanto las precisar la condición precaria y autoexplotada del trabajador o productor cultural-artístico. En tal sentido, dicha normalidad es claramente el efecto de la habitual neutralización de la resistencia crítica o la capacidad de contestación dentro de un espacio precarizado. De este modo, la normalización encubre la extrema tensión provocada por tendencias que se tornan dominantes pero que, paradojalmente, no corresponden necesariamente a una imposición ajena a la producción artística; así, el concepto de productividad normalizada pretende designar el proceso en el cual la colaboración auto-organizada de los agentes se somete voluntariamente a una resta de su potencia, al darse por horizonte los circuitos y las normas hegemónicas del valor.

2 Por otro lado, a partir de su estrecho vínculo con la conflictividad, el carácter excedente de la interacción y la cooperación puede ser asociado al rastro o la marca de la falla que es inherente y más bien constitutiva de lo social.

3 Esta línea de indagación desarrollada por un conjunto no menor de productores locales, marcó por ejemplo la presencia regional en la VI Bienal de Arte del Museo Nacional de Bellas Artes (2008). Bajo la curatoría de Simonetta Rossi, participaron en aquella versión los 
artistas Natascha de Cortillas, Carolina Maturana, Oscar Concha y MAS (Movimiento Artista del Sur).

4 Este perfil de trabajo fue diagramado en asociación con la Octava Mesa de Artes Visuales, agrupación que trabajó en la definición y elaboración de la convocatoria para exponer en la galería de Balmaceda Arte Joven, sede Biobío (2009).

\section{Referencias}

ROMERO, David. Soportes de inscripción contingente. Revista Plus, Soportes de Inscripción Contingente, Concepción, Chile, n. 1.

PANOTTO, Nicolás. Jacques Rancière y la política como desacuerdo I: la construcción del conflicto. Blog Gemrip, 7 jul. 2011. En internet: http://www.gemrip.com.ar/?p2

RANCIĖRE, Jacques. El viraje ético de la política y la estética. Santiago: Palinodia, 2005.

BISHOP, Claire. Antagonismo y estética relacional. Otra Parte, Revista de Letras y Artes, Buenos Aires, n. 5, 2005. En internet: http:// www.revistaotraparte.com/nº-5-otoño-2005/antagonismo-y-estética-relacional. 